\title{
An Attempt to Develop an Aptamer Lateral-Flow Assay (ALFA) for Easy, Rapid, and Sensitive Detection of Lethal Mushroom Toxin $\alpha$-amanitin
}

\author{
Qingchuan Chen ${ }^{1}$, Chen Fan ${ }^{1}$, Haozhe Huang ${ }^{1}$, Binglin $\mathrm{Xu}^{1}$, and Yeqing Zong ${ }^{2,}{ }^{*}$ \\ ${ }^{1}$ Shenzhen College of International Education, Antuoshan 6th Road, Futian District, \\ Shenzhen, Guangdong Province 518043, China \\ ${ }^{2}$ Bluepha Co., Ltd, Building A-F28, Daoxing Global Innovation Center, Liuxian 1st \\ Road, Baoan District, Shenzhen, Guangdong Province 518101, China \\ ${ }^{*}$ Correspondence should be addressed to Dr. Yeqing Zong, zongyeqing@bluepha.com
}

\begin{abstract}
Amatoxins contribute to the majority of mushroom poisoning, most prominently, $\alpha$-amanitin. Since mushroom is a common foodstuff worldwide, an easy, rapid, sensitive test for $\alpha$-amanitin is needed. Several detection methods for $\alpha$-amanitin have been developed, including HPLC, LC-MS, and ELISA, and LFIA. Aptamers have several advantages compared to antibodies: easy development via SELEX, longer shelf life, and higher temperature- and pH-tolerance. Aptamer Lateral Flow Assay (ALFA) is a similar technology compared to LFIA but incorporates aptamers as target-recognizing agents. This study attempted to develop an ALFA test strip for $\alpha$-amanitin using a previously-developed aptamer, however failure of generating a colorimetric readout at the test line is persisted throughout all experiments, even though the concept is fully-proved and the control line functions normally. The failure is attributed to the small size of the molecule, leading to immobilization difficulties on the nitrocellulose membrane to form the test line, and the hindering of effective "surround" mechanism of aptamer-target binding (instead of "adhere", when the target molecule is large, e.g. a protein). It is concluded that ALFAs for small-molecules whose aptamer-target interaction has not yet been studied and modelled in detail remains a challenge, despite ALFAs' large potential.
\end{abstract}

Keywords - $\alpha$-amanitin, Aptamer, Lateral Flow Assay

\section{Introduction}

Mushrooms have been a common foodstuff worldwide since civilization started. However, mushroom poisoning is one of the most serious food poisoning issues, with approximately one thousand cases reported globally each year. ${ }^{1-9}$. In 2020 , over 676 mushroom poisoning cases were reported in China alone, involving 1719 patients, with a death rate of $1.45 \%^{2}$. A large proportion of poisoning cases involved mushrooms containing amatoxins, and they also account for the majority of the fatalities ${ }^{1-3}$.

The main substances responsible for the toxicity of amatoxins are amanitins (AMAs), most prominently, $\alpha-, \beta-$, and $\gamma$-AMAs (Structures shown in Figure 1). Amanitins are reported to be potent inhibitors of RNA polymerase II, leading to fatal consequences of hindering protein synthesis in eukaryotes. ${ }^{10,11}$. Due to the stable cyclic structure of amanitins, they can neither be decomposed by heating (up to $100^{\circ} \mathrm{C}$ ) while the mushroom cooks, nor can they be degraded by enzymes in the organisms ${ }^{10}$. In addition, in humans, the symptoms of amanitin poisoning cannot be detected until 2 or 3 days after poisonous mushroom ingestion, and they are detected by renal or hepatic function test generally. However, amatoxin intoxication could lead to acute hepatic failure if the patient does not receive proper treatment within 3 to 4 days after ingestion ${ }^{12}$. Therefore, the need for a rapid, sensitive detection for amanitins is significant, either for hospital diagnosis or for detecting the mushroom itself.

Currently, laboratory detection of certain chemicals such as amatoxins is done by a number of laboratory-based methods which are highly dependent on heavy, intricate and expensive analytical devices, with complex sample preparation and result analysis steps that require skillful professionals ${ }^{13}$. 
High-Performance Liquid Chromatography (HPLC) ${ }^{14,15}$ and Liquid Chromatography-Mass Spectrometry (LC-MS) are two of these types of equipment. ${ }^{16,17}$. Aside from these, the Enzyme-Linked ImmunoSorbent Assay (ELISA) technique was also used to identify the presence of amatoxins and various chemical and biological targets. ${ }^{13}$. Although not dependent on expensive analytical equipment, ELISA still involves specialized reagents and multiple rounds of incubation and washing, and a test usually lasts for several hours. ELISA is still difficult to be carried out by non-experts and in non-laboratory environments. However, the concept of specific antibody-antigen binding was further applied in the Lateral-Flow ImmunoAssay (LFIA) technology ${ }^{18-22}$. Unlike in ELISA, all the reagents and processes are contained and undergone in a test strip - no washing or addition of reagents is required. Up to this day, LFIA is a well-established field ${ }^{19-22}$. Bever et al. developed the LFIA for $\alpha-, \beta-, \gamma$-AMAs ${ }^{18}$, and the whole procedure, including sample extraction, could be completed in less than a minute.

As a target-specific ligand, aptamers have several advantages in comparison to antibodies used in ELISA and LFIA. Aptamers are short RNA or single-stranded DNA (ssDNA) sequences usually of lengths 40-120 nucleotides and with unique secondary structures defined by their sequences. Through various types of intermolecular interactions ${ }^{23}$, aptamers can specifically bind with their designed targets, such as metal ions, peptides, proteins, or even cells ${ }^{24}$. Aptamers can be developed by a simple iterative in vitro selection process referred to as Systemic Evolution of Ligands by EXponential enrichment (SELEX) ${ }^{25,26}$ instead of being produced by immunized animals as in the case of antibodies ${ }^{27}$. They have longer shelf life and higher tolerance towards temperature and $\mathrm{pH}^{28}$.

Similar to antibodies in ELISA, aptamers could be adopted in a sensitive and selective test referred to as Enzyme-Linked OligoNucleotide Assay (ELONA), however the issue of requiring specialized reagents and costly equipment, and having a long duration persists ${ }^{29}$. Hence, based on a similar concept as LFIA, the Aptamer Lateral-Flow Assay (ALFA) technology has been developed and explored in recent years ${ }^{30-39}$. Targets of detection include dopamine ${ }^{31}$, thrombin ${ }^{40}$, Creatine Kinase MB $(\mathrm{CK}-\mathrm{MB})^{38}$, Human Epidermal Growth Factor Receptor 2 (HER2) ${ }^{39}$, and Escherichia Coli ${ }^{41}$. The most common formats of ALFA are sandwich and competitive. In the sandwich format, the target-specific aptamer is both conjugated to reporting agents (such as gold nanoparticles (AuNP) for an observable red color or quantum dots (QD) for a measurable photoluminescence) and immobilized on the test line, allowing them to hold the target molecule (if present) in the middle, forming a "sandwich" structure ${ }^{40,41}$. In the competitive format, the test line is composed of immobilized target molecules, competing with the target molecule present in the analyte for binding with the reporter aptamer probe ${ }^{30,42}$. In either format, the reporting intensity is positively correlated to the amount of reporter aptamer probe bound on the test line. But due to differences in their functioning mechanisms, the reporting intensity of the former positively correlates to the target molecule concentration, while that of the latter, negatively. But in both cases, the control line is designed to be able to capture the reporter aptamer probe, no matter if it is

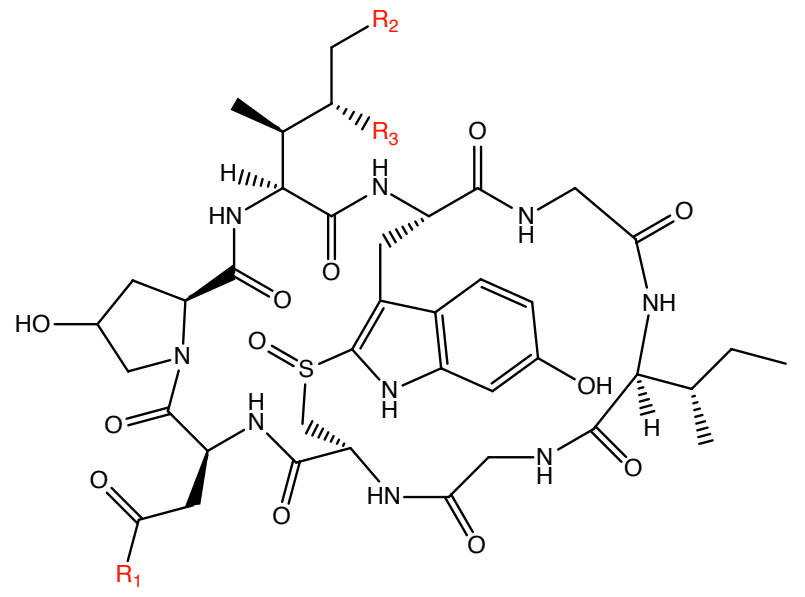

\begin{tabular}{cccc}
\hline Subtype & $\mathbf{R}_{\mathbf{1}}$ & $\mathbf{R}_{\mathbf{2}}$ & $\mathbf{R}_{\mathbf{3}}$ \\
\hline $\mathrm{a}$ & $\mathrm{NH}_{2}$ & $\mathrm{OH}$ & $\mathrm{OH}$ \\
\hline$\beta$ & $\mathrm{OH}$ & $\mathrm{OH}$ & $\mathrm{OH}$ \\
\hline $\mathrm{Y}$ & $\mathrm{NH}_{2}$ & $\mathrm{OH}$ & $\mathrm{H}$ \\
\hline
\end{tabular}

Figure 1: The chemical structures of $\alpha-\beta$-, and $\gamma$-amanitins. 
bioRxiv preprint doi: https://doi.org/10.1101/2021.09.27.461950; this version posted September 27, 2021. The copyright holder for this preprint (which was not certified by peer review) is the author/funder, who has granted bioRxiv a license to display the preprint in perpetuity. It is made available under aCC-BY 4.0 International license.

\begin{tabular}{cc}
\hline Name & ssDNA Sequence \\
\hline Biotin-Apt & 5'-Biotin-C6-catgcttcccagggagatggaggtctttttggttgttggtgggggaatcttttggtattgaggaacatgcgtcgcaaac-3' \\
Thiol-Apt & 5'-Thiol-C6-catgcttcccagggagatggaggtctttttggttgttggtgggggaatcttttggtattgaggaacatgcgtcgcaaac-3' \\
Biotin-cApt & 5'-Biotin-C6-gtttgcgacgcatgttcctcaataccaaaagattccccaccaacaaccaaaaagacctccatctccctggggaagcatg-3' \\
\hline
\end{tabular}

Table 1: The names and ssDNA sequences for the (modified) aptamers and complementary sequences used in this research.

bound to the target molecule, either by a poly-A strand binding on the reporter aptamer ${ }^{40}$, an antibody which binds to an antigen conjugated with the aptamer probe ${ }^{41}$, or a complete complementary sequence to the reporter aptamer that would displace the aptamer from the complex with the target molecule ${ }^{30}$.

In this study, we report an attempt to develop a competitive ALFA test for the lethal mushroom toxin present in Amanita phalloides, $\alpha$-amanitin ( $\alpha$-AMA), using the aptamer developed by Muszyńska et al. ${ }^{43}$. Although we have validated the specificity of aptamer-target binding and the competitive binding between sample toxin molecules and immobilized toxin molecules necessary for the functioning of the test line by a novel approach referred to as Magnetic Beads-ELONA (MB-ELONA), the test strip development was consistently unsuccessful. An analysis of the possible reasons for this failure is provided.

\section{Materials and Methods}

\section{$2.1 \quad$ Materials}

Sodium periodate $\left(\mathrm{NaIO}_{4}\right)$, Sodium cyanoborohydride $\left(\mathrm{NaBH}_{3} \mathrm{CN}\right)$, Tris $(2$-carboxyethyl)phosphine hydrochloride (TCEP HCl), Tween-20, BSA (Bovine Serum Albumin), TMB (3,3',5,5'-tetramethylbenzidine) chromogenic substrate, $\alpha$-AMA, and all other reagents, if not stated otherwise, are purchased from Sigma-Aldrich. Test strip components, including plastic test strip shells, backing cards, absorbent pads, and nitrocellulose membranes were purchased from Jieyi Biotech (Shanghai, China). Carboxyl Magnetic Bead Coupling Kit was purchased from Sangon (Shanghai, China). Biotinylated and thiolated ssDNA aptamers (and some complementary sequences) for $\alpha$-AMA, whose sequences are derived from the $\alpha$-AMA aptamer developed by Muszyńska et al. ${ }^{43}$ were synthesized by a commercial DNA synthesis company, with their respective sequences tabulated in Table 1. Colloidal gold nanoparticles $(10-20 \mathrm{~nm})$ solution was purchased from XFNano (Jiangsu, China).

\subsection{Buffer solutions}

The 1x PBS (10 mM phosphate, $138 \mathrm{mM} \mathrm{NaCl}, 2.7 \mathrm{mM} \mathrm{KCl}, \mathrm{pH} 7.4)$ is prepared by diluting the stock 10x PBS solution. The pH 9.6 Carbonate Buffer Solution (CBS) is 0.05 M carbonate-bicarbonate buffer solution. The Binding Buffer Solution (BBS) is prepared by dissolving $\mathrm{MgCl}_{2}$ into $1 \mathrm{x}$ PBS until a final concentration of $2.5 \mathrm{mM}$ (for $\mathrm{MgCl}_{2}$ ) is achieved. The PBS-Tween (PBST) solution is prepared by adding $0.1 \% \mathrm{v} / \mathrm{v}$ Tween-20 into $1 \mathrm{x}$ PBS.

\subsection{Conjugation of $\alpha$-AMA with Bovine Serum Albumin (BSA)}

The conjugation between $\alpha$-AMA and Bovine Serum Albumin (BSA) is performed according to a previously-reported experimental protocol ${ }^{44}$. An aqueous solution of $\alpha$-AMA $(500 \mu \mathrm{l}, 2 \mathrm{mg} / \mathrm{ml})$ is first activated upon addition of $\mathrm{NaIO}_{4}$ solution $(218 \mu \mathrm{l}, 40 \mathrm{mM})$ and stirring under room temperature in dark for 20 minutes. The mixture is then added to $615 \mu \mathrm{l}$ BSA solution $(10 \mathrm{mg} / \mathrm{ml}$, in pH $9.6 \mathrm{CBS})$, then put under room temperature in dark conditions for 2 hours with tilt rotation. Finally, $51 \mu \mathrm{l} \mathrm{NaBH}_{3} \mathrm{CN}$ solution $(0.1 \mathrm{M}$, in $10 \mathrm{mM} \mathrm{NaOH}(\mathrm{aq}))$ is added to the mixture, and stirred under room temperature in dark for another 2 hours.

\subsection{Conjugation of proteins ( $\alpha$-AMA-BSA and BSA) with MB}

The conjugation is done using the reagents and according to the instructions from the Carboxyl Magnetic Bead Coupling Kit from Sangon (Shanghai, China). Briefly, 100 $\mu$ magnetic beads are activated by an incubation of 15 minutes with sulfo-NHS $(50 \mathrm{mg} / \mathrm{ml})$ and EDC solutions $(50 \mathrm{mg} / \mathrm{ml})$ prepared using MES buffer, and are separated from the mixture using a neodymium magnet. The beads are then incubated with $0.5 \mathrm{mg} \alpha$-AMA-BSA along with conjugation buffer (PBS) for 1.5 hours under room temperature, 
bioRxiv preprint doi: https://doi.org/10.1101/2021.09.27.461950; this version posted September 27, 2021. The copyright holder for this

followed by addition of blocking buffer (BSA solution in PBS) for 30 min. After several further addition and washing with blocking buffer and preservation buffer $\left(0.02 \% \mathrm{NaN}_{3}\right.$ solution in PBS $)$, the final product is resuspended in preservation buffer and stored at $4^{\circ} \mathrm{C}$.

\subsection{Magnetic Beads-ELONA (MB-ELONA) for the validation of principle for the competitive test strip}

The $\alpha$-AMA-BSA-MB is thoroughly mixed by vortex, and six groups of the magnetic beads solution $(5 \mu \mathrm{l})$ are dispensed into separate Eppendorf microcentrifuge tubes. A neodymium magnet is used to attract and immobilize the magnetic beads onto one side of the tube, and the supernatant is decanted. $250 \mu \mathrm{l}$ biotin-Apt (diluted to $0.75 \mu \mathrm{M}$ using BBS) is heated in a metal bath under $90^{\circ} \mathrm{C}$ for 5 minutes and immediately inserted into ice to allow the formation of tertiary structures (folding) of the ssDNA aptamer. The aptamer solution is then separated into five equal portions, $50 \mu \mathrm{l}$ each. Aqueous $\alpha$-AMA solution $(5 \mu \mathrm{l})$ of concentrations $10 \mu \mathrm{g} / \mathrm{ml}, 1 \mu \mathrm{g} / \mathrm{ml}, 100 \mathrm{ng} / \mathrm{ml}, 10 \mathrm{ng} / \mathrm{ml}$, and $0 \mathrm{ng} / \mathrm{ml}$ are added to the five aptamer solutions, one concentration per group. These mixtures are then added to five of the six groups of $\alpha$-AMA-BSA-MB, respectively. For the last group (background group), $55 \mu \mathrm{L}$ of BBS is added. The solutions are incubated for 15 minutes under room temperature, under stirring conditions (alternatively, the mixtures are vortexed every several minutes).

After incubation, the magnetic beads are separated from the solution and immobilized by the neodymium magnet, and the supernatant is decanted. The magnet is removed, and $50 \mu \mathrm{PBST}$ is added to each microcentrifuge tube, which are then vortexed to mix thoroughly. The magnetic beads are once again separated and immobilized using the magnet, and the supernatant is decanted. This washing process is repeated 5 times. After washing, the magnetic beads are incubated with $100 \mu \mathrm{L} 1 / 2000 \mathrm{v} / \mathrm{v}$ Streptavidin-Horseradish Peroxidase (SA-HRP) solution for 15 minutes under room temperature with stirring/vortexing, and washed 5 times with PBST after supernatant decantation. Finally, $50 \mu \mathrm{l}$ of the TMB chromogenic substrate solution is added to each of the microcentrifuge tubes and thoroughly mixed by vortex, and are incubated under $37^{\circ} \mathrm{C}$ in the dark for 15 minutes, with stirring/vortexing. Magnetic beads are once again separated and immobilized using a magnet, and the supernatant is transferred into a 96-well microtitre plate, and the reaction is stopped upon adding $25 \mu$ l stopping buffer $\left(2 \mathrm{M} \mathrm{H}_{2} \mathrm{SO}_{4}(\mathrm{aq})\right)$. The absorbance at wavelength $450 \mathrm{~nm}\left(\mathrm{OD}_{450}\right)$ of the resulting solutions are measured.

Three control groups are also set up to confirm the specificity of the aptamer binding. The $\alpha$-AMABSA-MB are replaced with BSA-MB. Two groups $(10 \mu \mathrm{g} / \mathrm{ml}$ and $0 \mu \mathrm{g} / \mathrm{ml}$ alpha-AMA) and one control group (background group) of the MB-ELONA experiment are undergone through the same procedures as above.

\subsection{Preparation of lateral flow test strips}

All test strip components are cut with width of $3 \mathrm{~mm}$. A $45 \mathrm{~mm}$ long strip of nitrocellulose membrane and a $17 \mathrm{~mm}$ long strip of absorbent pad are cut.

For the test line, a 1:1 volume ratio of $2 \mathrm{mg} / \mathrm{ml} \alpha$-AMA aqueous solution is dotted onto the test strip, $30 \mathrm{~mm}$ from the start of the nitrocellulose membrane, using a 0.2-20 $\mu$ l Eppendorf tip as 4 consecutive dots that connects to form a line. When the line is completely dried, $1 \mathrm{mg} / \mathrm{ml} \mathrm{SA}$ solution is dotted onto the same position using the same method.

For the control line, a solution of biotin-Apt is pre-incubated with SA solution at a molar ratio of $3: 1$, at room temperature for 1 hour, and the miture is dispensed onto the membrane through the same method as the test line, but $35 \mathrm{~mm}$ from the start. The membrane is left dry completely under room temperature, and $5 \% \mathrm{w} / \mathrm{v}$ BSA in PBST is added to the membranes as a blocking agent. The membranes are then dried completely under $50^{\circ} \mathrm{C}$. After drying, all the test strip components are assembled onto a $60 \mathrm{~mm}$ long adhesive backing card, with $2 \mathrm{~mm}$ overlap between the nitrocellulose membrane and the absorbent pad.

\subsection{Preparation of AuNP-Apt conjugates}

The experimental protocol for the functionalization of AuNP with thiol-Apt is derived and adapted from that reported in the work of Dalirirad et al. ${ }^{31}$. $2.5 \mu \mathrm{l}$ of $100 \mu \mathrm{M}$ Thiol-Apt is added to $22.5 \mu \mathrm{l}$ BBS and vortexed to mix thoroughly. The mixture is heated to $90^{\circ} \mathrm{C}$ on metal bath for 5 minutes, and then immediately inserted into ice. $1 \mu \mathrm{l}$ of $20 \mathrm{mM}$ aqueous TCEP solution is added to the aptamer solution, 
mixed thoroughly, and incubated at room temperature for 1 hour to allow reduction of disulfide bonds between the commercially-synthesized thiol-Apt and the liberation of free thiols.

$20 \mu \mathrm{l}$ of the above activated aptamer solution is added into $400 \mu \mathrm{l}$ colloidal AuNP solution (centrifuged and concentrated to calibrate to $\left.\mathrm{OD}_{520}=7.5\right)$. and mixed thoroughly. The mixture is incubated without light over night ( $\geq 8$ hours). Then, to the mixture, $105 \mu$ of $1 \mathrm{M}$ aqueous $\mathrm{NaCl}$ solution is added for the aging process $(10 \mu \mathrm{l}$ ever 20 minutes, except for the last addition, which is $5 \mu$ l only). After each addition, the solution is carefully and gently mixed by vortex, and constantly stirred by tilt rotation. The mixture is then put under $4^{\circ} \mathrm{C}$ without light for 24 hours.

Then, the AuNP-Apt conjugate solution is centrifuged (12500 rpm, 15 minutes), and the supernatant is decanted. $400 \mu \mathrm{l}$ deionized water is added, mixed well, and then centrifuged (12500 rpm, 15 minutes) followed by supernatant decantation. The washing process is then repeated twice in addition ( 3 rounds in total), and the washed conjugate is resuspended in $400 \mu \mathrm{l}$ deionized water.

\subsection{Aptamer Lateral Flow Assays (ALFA)}

$15 \mu \mathrm{l}$ of AuNP-Apt conjugate is mixed thoroughly with $15 \mu \mathrm{l}$ sample solution, and is dispensed onto the test strip. A colorimetric readout is obtained after 5 minutes, when all the conjugate has flown over the test and control lines.

When the ALFA tests are completed, the test strips are dried under room conditions, and a smartphone built-in digital camera is used to take pictures of the test strips. The images are then desaturated using the Adobe Photoshop software, and 10 random points from each test/control line area are selected to take brightness values. The mean values and standard deviations are then calculated.

\section{Results and Discussion}

\subsection{Conjugation of $\alpha$-AMA with BSA}

The purpose of conjugation of $\alpha$-AMA with BSA is to indirectly conjugate $\alpha$-AMA onto the carboxyl magnetic bead $(\mathrm{MB})$. The carboxyl moieties $\left(-\mathrm{CO}_{2} \mathrm{H}\right)$ on its surface can undergo condensation reactions with primary amine $\left(-\mathrm{NH}_{2}\right)$ groups on other molecules, under the activating agents EDC and sulfoNHS. However, the $\alpha$-AMA itself has no primary amine groups available for the condensation reaction. Instead, BSA is used as the intermediate which bridges between the $\alpha$-AMA and the MB. BSA has the primary amine groups required for condensation with $\mathrm{MB}$ and can also be conjugated with $\alpha$-AMA using
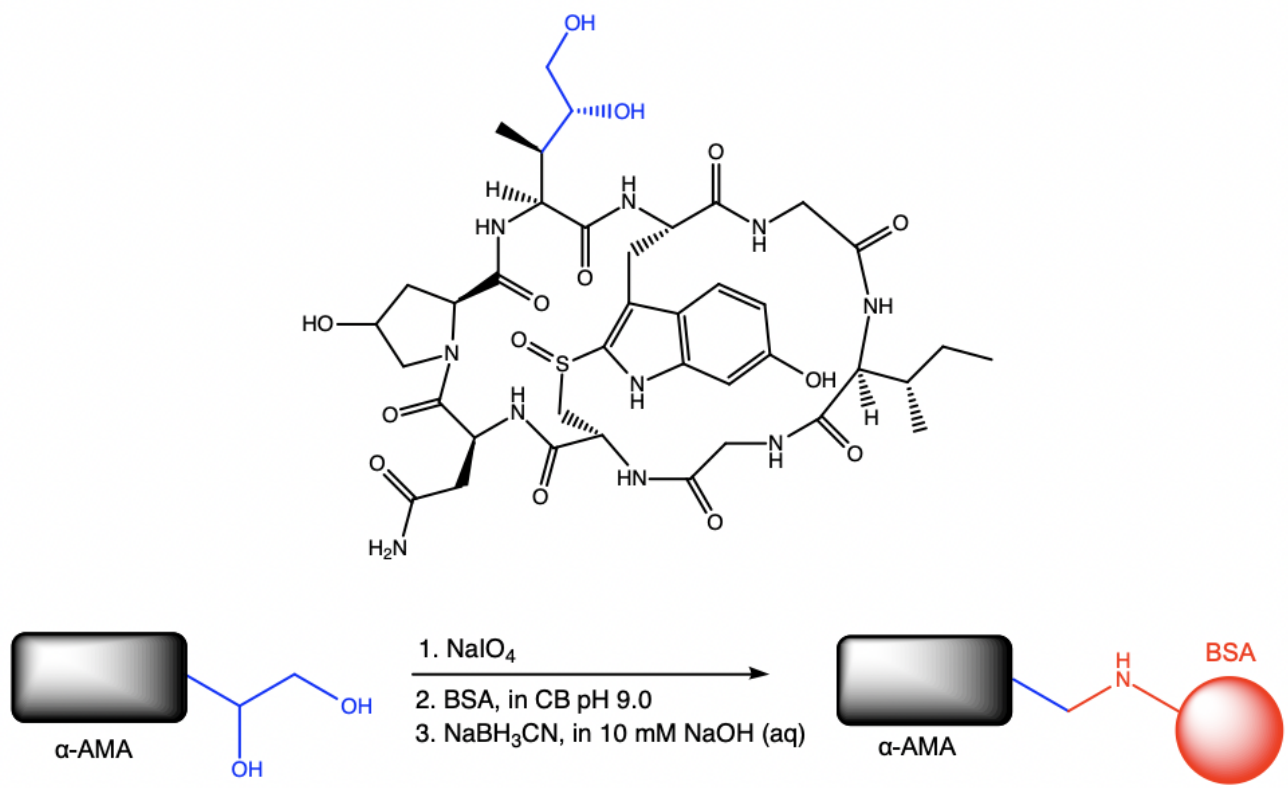

Figure 2: A schematic representation of the conjugation between $\alpha$-AMA with BSA, whose method is devised by Bever et al. ${ }^{44}$. 
the method stated in Section 2.3 (Figure 2). Briefly, the 1,2-diol on $\alpha$-AMA is oxidized by periodate to an aldehyde, which then undergoes carbonyl-amine condensation with primary amine groups on BSA. The resulted imine is prone to undergo hydrolysis, and hence is reduced to the more stable secondary amine by a mild reducing agent $\mathrm{NaBH}_{3} \mathrm{CN}$. The remaining primary amine groups on BSA unoccupied by $\alpha$-AMA are available for the conjugation to carboxyl MB.

\subsection{Competitive MB-ELONA experiments to validate the working principles of the test line}

Competitive MB-ELONA is used to prove the concept of competitive binding of the aptamer onto the free $\alpha$-AMA molecules in the sample solution and immobilized $\alpha$-AMA molecules on the nitrocellulose membrane. The aptamer is first incubated with different concentrations of $\alpha$-AMA. As concentration of $\alpha$-AMA decreases, the amount of free aptamer left unbound in the solution increases, so that more of which will bind to the $\alpha$-AMA moieties on the $\alpha$-AMA-BSA-MB. followed by fixing of SA-HRP, resulting in a higher rate of reaction when TMB solution is added. This is demonstrated by a distinct increase in $\mathrm{OD}_{450}$ as concentration of free $\alpha$-AMA decreases from $10 \mu \mathrm{g} / \mathrm{ml}$ to $0 \mathrm{ng} / \mathrm{ml}$ (Figure 3 (iii)).

Compared to the conventional ELONA, MB-ELONA has several notable advantages. Firstly, because the solid phase in ELONA is the bottom and the walls of wells in 96-well microtitre plates, while that in MB-ELONA is the nanometer-scale magnetic beads, the surface area of contact between the solid phase and the solution phase is significantly larger in MB-ELONA. Combined with the ability to continuously stir and mix the magnetic beads suspension in order to maximize contact, the reaction kinetics in MB-ELONA is considerably faster. Hence, the duration of incubation procedures (with biotinylated aptamers and SA-HRP) in MB-ELONA can be reduced down to 15 minutes (in this research) or possibly even further, compared to up to 1 hour in conventional ELONA experiments ${ }^{29}$. Secondly, because target molecules are covalently bonded to magnetic beads (while in ELONA they are coated onto the microtitre plate through electrostatic or other types of weaker interactions), the possibility of target molecules detaching from the solid phase in washing steps is reduced, and washing steps can be much more easily achieved since all magnetic beads can be completely separated from solution with the aid of a bar of magnet. Although the preparation of target molecule-coated solid phase is less experimentally straightforward in MB-ELONA (requiring a pure sample of the target molecule for conjugation), as opposed to a simple coating-washing-blocking procedure (of any sample solution awaiting analysis) in ELONA, a competitive MB-ELONA test, like the one described in this research, could be used, in which a sample solution is added as solution phase in the aptamer incubation step, with any possibly-existing target molecules competing for aptamer binding against the pure target molecule immobilized on the magnetic beads.

\subsection{Competitive Aptamer Lateral-Flow Assays (ALFA)}

A diagram of the ALFA test strips assembled according to the experimental procedure mentioned above is provided in Figure 4. The results are shown in Figure 5.

For the control line, according to the displacement principle, the higher affinity of the aptamer to its complementary strands than to $\alpha$-AMA will always result in a displacement of AuNP-Apt and the binding of which to $\alpha$-AMA-cApt on the control line, so that all aptamer, free or bound, will bind to the complementary strands on the control line, which is demonstrated by an unchanged brightness of the control line despite of the different concentrations of $\alpha$-AMA ranging from $100 \mu \mathrm{g} / \mathrm{ml}$ to $0 \mu \mathrm{g} / \mathrm{ml}$ (Figures 5 (ii)).

However, the ALFA tests have consistently lead to a failure in AuNP aggregation on the test line. In all tests, the difference in brightness values at the test line is insignificant from the rest of the nitrocellulose membrane, and no observable colorimetric readout could be obtained.

The successful results in MB-ELONA verifies that there exists a target-specific binding between the aptamer and the $\alpha$-AMA, and a competition between the aptamer's binding to the free solution-phase and the immobilized-phase $\alpha$-AMA molecules could be clearly identified. Hence the failure of AuNP aggregation could be attributed to either:

1. The small size of the $\alpha$-AMA, resulting in failure to be immobilized onto the nitrocellulose membrane, and is easily carried away from the test line by the movement of the sample liquid;

2. The prohibition of effective binding between aptamer and $\alpha$-AMA due to the complex environment made by the fibrilic structure within the nitrocellulose membrane. 
(i)

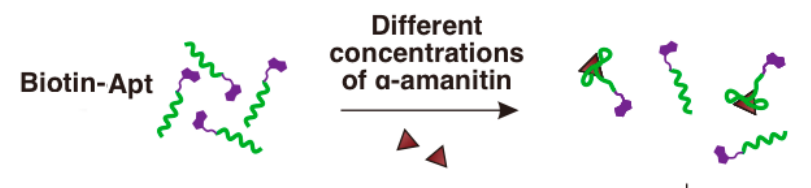

(ii)
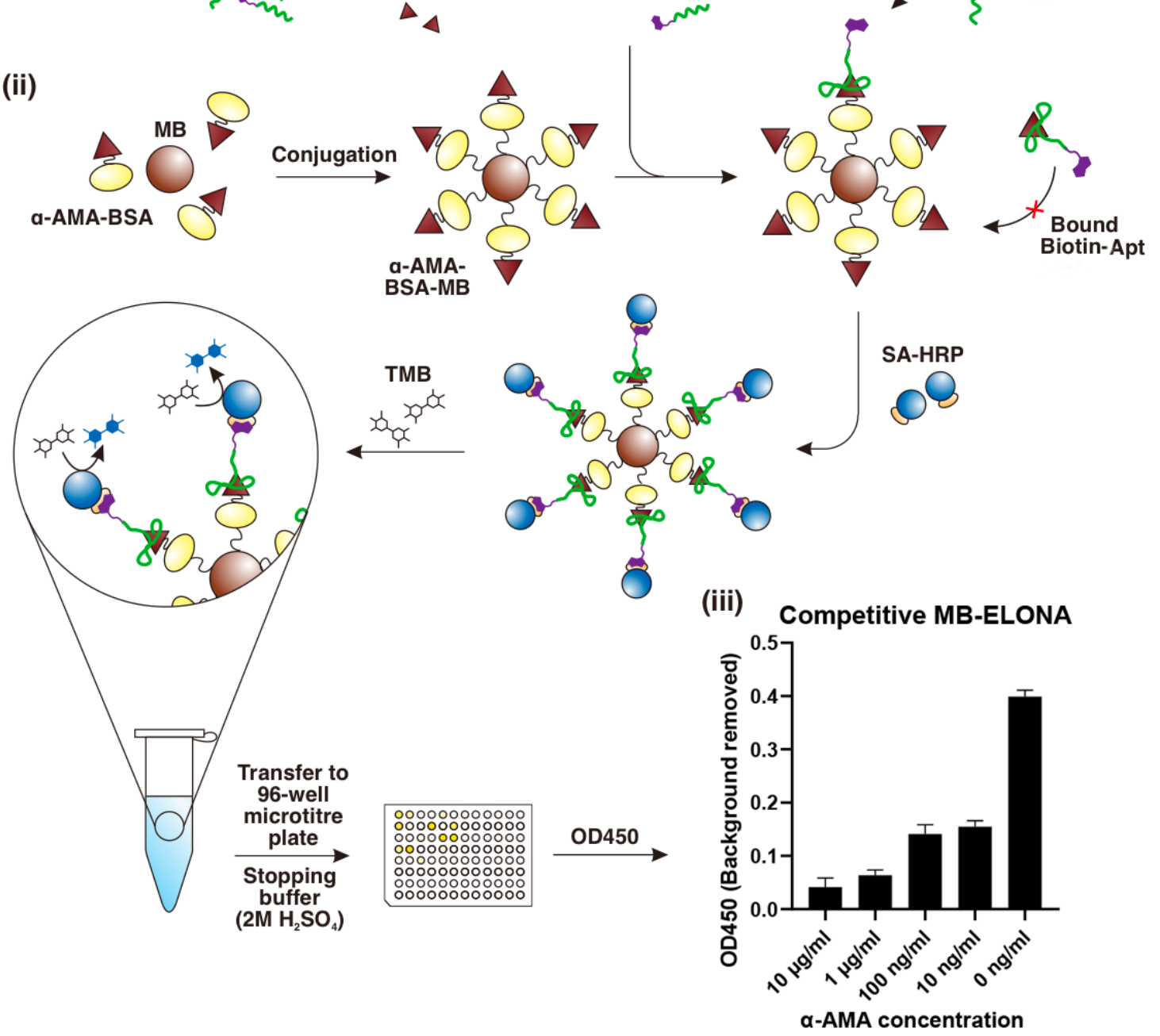

Figure 3: (i) A schematic representation of the preincubation of Biotin-Apt with different concentrations of free $\alpha$-AMA, (ii) a schematic representation of the competitive MB-ELONA experiment, and (iii) the results of the competitive MB-ELONA. 
bioRxiv preprint doi: https://doi.org/10.1101/2021.09.27.461950; this version posted September 27, 2021. The copyright holder for this preprint (which was not certified by peer review) is the author/funder, who has granted bioRxiv a license to display the preprint in perpetuity. It is made available under aCC-BY 4.0 International license.

(i)

Loading position of sample + gold nanoparticle-aptamer mixture

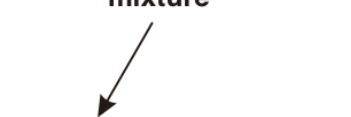

\section{Nitrocellulose}

membrane

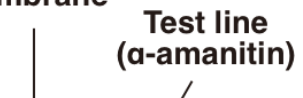

\section{Control line}

(SA-Biotin-cApt)
Absorbent pad

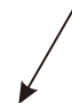

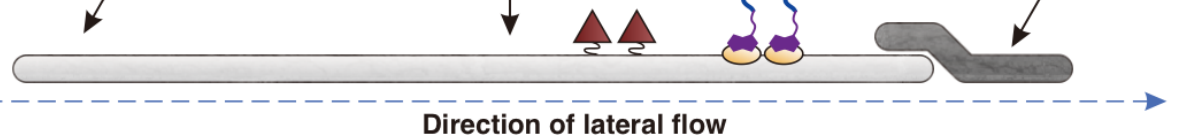

(ii)

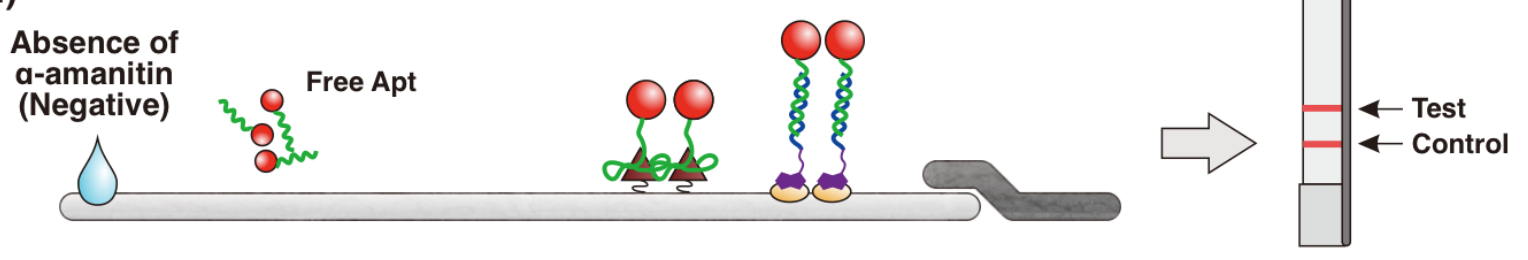

(iii)

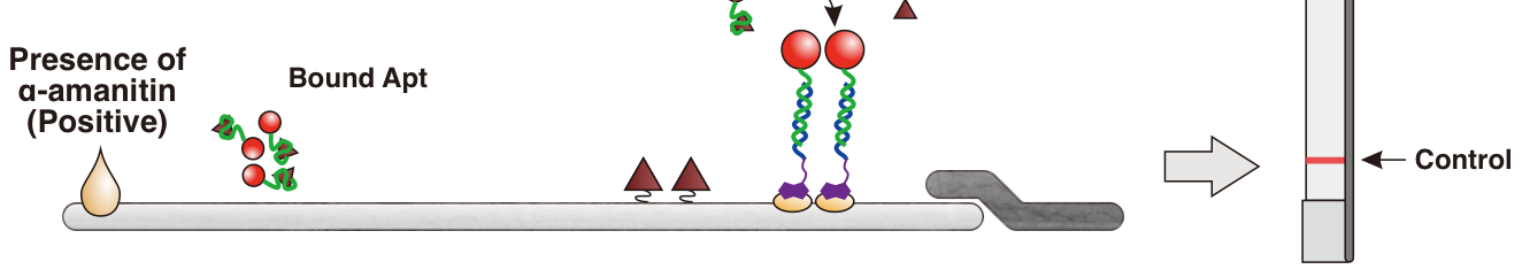

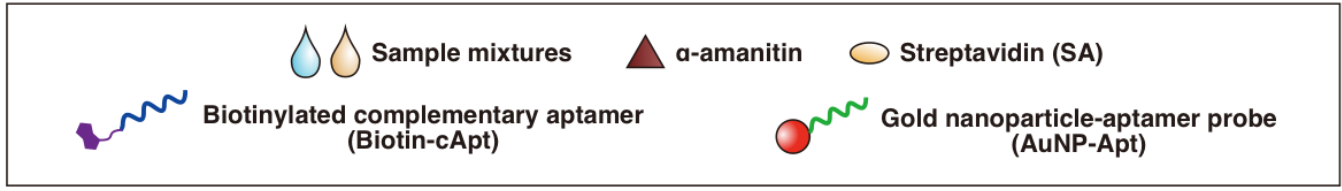

Figure 4: A representative diagram of the (i) structure of the ALFA test strip, (ii) the $\alpha$-AMA-negative ALFA test, and (iii) the $\alpha$-AMA-positive ALFA test.

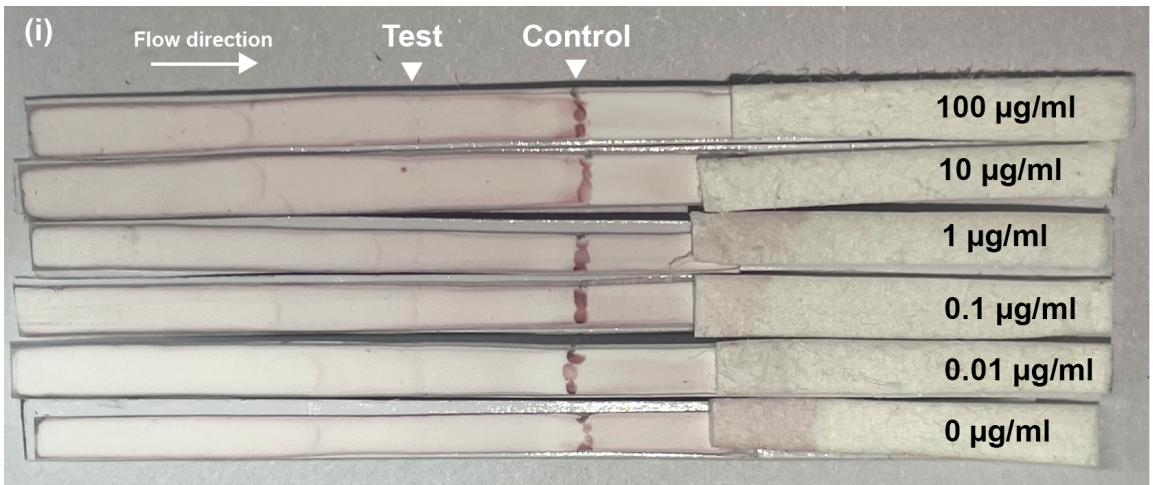

(ii)

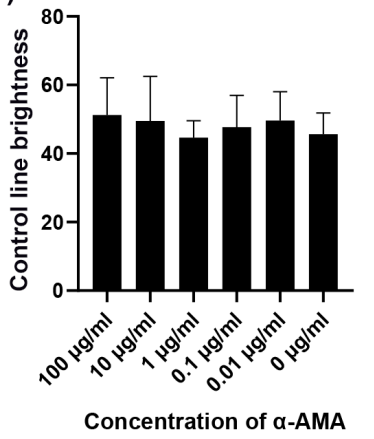

Figure 5: (i) A labelled image, taken by a smartphone built-in camera, of the ALFA test strips, when sample liquids containing different concentrations of $\alpha$-AMA are dropped onto the strips, and (ii) the brightness values of the control lines taken from the image using Adobe Photoshop. 
Initially, reason 1 may seem unreasonable as a failure in AuNP aggregation is persisted when the test line solution is switched to the $\alpha$-AMA-BSA conjugate. It would have been reasonable to suggest that the large size of the BSA protein reduces the possibility for the weak immobilization of the toxin molecule, and it could be no longer carried away by the moving sample liquid. However, concerns on the efficiency and affinity of binding between the aptamer and the chemically-modified $\alpha$-AMA, conjugated with BSA, were raised. Although the work by Bever et al. ${ }^{18}$ demonstrated that such conjugate was still suitable for antibody binding, it is not necessarily so for aptamers due to differences in binding mechanisms.

Reason 2 is logically justified, because in contrast to on the nitrocellulose membrane, the MB-ELONA experiments succeeded as the aptamer binds to the immobilized target in the unhindered solution phase. Due to the small size of the $\alpha$-AMA molecule, it is assumed that the aptamer adopts a "surround" binding mechanism, as a loop formed by the secondary structure of the ssDNA binds to the $\alpha$-AMA, similar to a chelation reaction. In other previous ALFA studies, where larger target molecules (often proteins) were used, for instance, HER2 $2^{39}$ and thrombin ${ }^{40}$, aggregation of AuNP on the test line has yielded success, as the aptamers in these studies adopted an "adhere" binding mechanism, where the aptamers fit into external characteristic sites of proteins, not involving the formation of loops and surrounding around targets. It might be concluded that the adhere mechanism of aptamer-target binding is more effective and robust in complex environments like on nitrocellulose membranes compared to the surround mechanism.

However, in some other ALFA researches, where the target molecule is even smaller and the aptamertarget binding mechanism is well-studied, for instance, detection of dopamine in urine ${ }^{37}$, a stranddisassociation design of the ALFA test could be adopted when a known and well-modelled morphological change in the aptamer is induced upon its binding with the target molecule, causing it to unwind from a short segment of its countersequence, covalently bonded onto AuNP. The exposure of the bare countersequence on the AuNP allows it to bind to another portion of the aptamer sequence, immobilized on the test line, causing AuNP aggregation, generating a colorimetric readout. However, this could not yet be applied to $\alpha$-AMA ALFA as the binding mechanism of the aptamer used in this study developed by Muszyńka et al. ${ }^{43}$ awaits further detailed investigations.

\section{Conclusion}

Despite the sufficient specificity and affinity of the $\alpha$-AMA aptamer used and the successful verification of competitive binding principle for the functioning of the test line as well as strand displacement principle for the control line, it was comprehensively unsuccessful in the development of a fully functioning ALFA test strip for $\alpha$-AMA in this research. The reasons for this failure were briefly analysed in previous sections: it was believed that the small size of $\alpha$-AMA prohibited its immobilisation onto the nitrocellulose membrane, or that the binding of aptamer with $\alpha$-AMA within the nitrocellulose membrane was not achieved due to the complex structure of the membrane. It resides a challenge in the development of ALFA test strips when the detailed binding mechanism and interactions between aptamer and its target is unstudied, especially if the target is comparatively a small-molecule, and the aptamer binds to the target via the "surround" mechanism instead of the "adhere" mechanism. However, ALFA is still a prospective field of study with a large potential, and this research has provided it with new insights, discussions and a new method of MB-ELONA.

\section{Acknowledgements}

The authors of this study acknowledge Bluepha Co., Ltd for providing the laboratory site and equipments.

\section{References}

[1] Haijiao Li, Hongshun Zhang, Yizhe Zhang, Kaiping Zhang, Jing Zhou, Yu Yin, Shaofeng Jiang, Peibin Ma, Qian He, Yutao Zhang, et al. Mushroom poisoning outbreaks - china, 2019. China CDC Weekly, 2(2):19-24, 2020.

[2] Haijiao Li, Hongshun Zhang, Yizhe Zhang, Jing Zhou, Yu Yin, Qian He, SHaofeng Jiang, Peibin Ma, Yutao Zhang, Ke Wen, Yuan Yuan, Nan Lang, Bowen Cheng, Junjia Lu, and Chengye Sun. Mushroom poisoning outbreaks — china, 2020. China CDC Weekly, 3(3):41, 2021. 
[3] William E Brandenburg and Karlee J Ward. Mushroom poisoning epidemiology in the united states. Mycologia, 110(4):637-641, 2018.

[4] David D Gummin, James B Mowry, Michael C Beuhler, Daniel A Spyker, Daniel E Brooks, Katherine W Dibert, Laura J Rivers, Nathaniel PT Pham, and Mark L Ryan. 2019 annual report of the american association of poison control centers' national poison data system (npds): 37 th annual report. Clinical Toxicology, 58(12):1360-1541, 2020.

[5] David D Gummin, James B Mowry, Daniel A Spyker, Daniel E Brooks, Michael C Beuhler, Laura J Rivers, Heba A Hashem, and Mark L Ryan. 2018 annual report of the american association of poison control centers' national poison data system (npds): 36th annual report. Clinical toxicology, 57(12):1220-1413, 2019.

[6] David D Gummin, James B Mowry, Daniel A Spyker, Daniel E Brooks, Krista M Osterthaler, and William Banner. 2017 annual report of the american association of poison control centers' national poison data system (npds): 35th annual report. Clinical toxicology, 56(12):1213-1415, 2018.

[7] Sevki Hakan Eren, Yeltekin Demirel, Serdal Ugurlu, Ilhan Korkmaz, Can Aktas, and Fatma Mutlu Kukul Güven. Mushroom poisoning: retrospective analysis of 294 cases. Clinics, 65(5):491496, 2010.

[8] G Cervellin, I Comelli, G Rastelli, F Sanchis-Gomar, F Negri, C De Luca, and G Lippi. Epidemiology and clinics of mushroom poisoning in northern italy: A 21-year retrospective analysis. Human $\&$ experimental toxicology, 37(7):697-703, 2018.

[9] Juliana Garcia, Vera M Costa, Alexandra Carvalho, Paula Baptista, Paula Guedes de Pinho, Maria de Lourdes Bastos, and FÚlix Carvalho. Amanita phalloides poisoning: Mechanisms of toxicity and treatment. Food and chemical toxicology, 86:41-55, 2015.

[10] Theodor Wieland, Heinz Faulstich, and Luigi Fiume. Amatoxins, phallotoxins, phallolysin, and antamanide: the biologically active components of poisonous amanita mushroom. CRC critical reviews in biochemistry, 5(3):185-260, 1978.

[11] MS Bonnet and PW Basson. The toxicology of amanita phalloides. Homeopathy, 91(4):249-254, 2002.

[12] Michael R Smith and Robert L Davis. Mycetismus: a review. Gastroenterology report, 4(2):107-112, 2016.

[13] Candace S Bever, Robert M Hnasko, Luisa W Cheng, and Larry H Stanker. A rapid extraction method combined with a monoclonal antibody-based immunoassay for the detection of amatoxins. Toxins, 11(12):724, 2019.

[14] Flavio Belliardo and Graziella Massano. Determination of $\alpha$-amanitin in serum by hplc. Journal of Liquid Chromatography, 6(3):551-558, 1983.

[15] F Jehl, C Gallion, P Birckel, A Jaeger, F Flesch, and R Minck. Determination of $\alpha$-amanitin and $\beta$-amanitin in human biological fluids by high-performance liquid chromatography. Analytical biochemistry, 149(1):35-42, 1985.

[16] Thomas Gicquel, Sylvie Lepage, Manon Fradin, Olivier Tribut, Benedicte Duretz, and Isabelle Morel. Amatoxins ( $\alpha$-and $\beta$-amanitin) and phallotoxin (phalloidin) analyses in urines using highresolution accurate mass lc-ms technology. Journal of analytical toxicology, 38(6):335-340, 2014.

[17] Nicole L Abbott, Kasey L Hill, Alaine Garrett, Melissa D Carter, Elizabeth I Hamelin, and Rudolph C Johnson. Detection of $\alpha-, \beta$-, and $\gamma$-amanitin in urine by lc-ms/ms using $15 \mathrm{n} 10-\alpha-$ amanitin as the internal standard. Toxicon, 152:71-77, 2018.

[18] Candace S Bever, Catharine A Adams, Robert M Hnasko, Luisa W Cheng, and Larry H Stanker. Lateral flow immunoassay (lfia) for the detection of lethal amatoxins from mushrooms. PloS one, 15(4):e0231781, 2020.

[19] Kathryn H Ching, Alice Lin, Jeffery A McGarvey, Larry H Stanker, and Robert Hnasko. Rapid and selective detection of botulinum neurotoxin serotype-a and-b with a single immunochromatographic test strip. Journal of immunological methods, 380(1-2):23-29, 2012. 
[20] Der-Jiang Chiao, Rong-Hwa Shyu, Chieh-Shen Hu, Hsien-Yuan Chiang, and Shiao-Shek Tang. Colloidal gold-based immunochromatographic assay for detection of botulinum neurotoxin type b. Journal of Chromatography B, 809(1):37-41, 2004.

[21] Shyu Rong-Hwa, Tang Shiao-Shek, Chiao Der-Jiang, and Hung Yao-Wen. Gold nanoparticle-based lateral flow assay for detection of staphylococcal enterotoxin b. Food Chemistry, 118(2):462-466, 2010.

[22] Eiki Yamasaki, Ryuta Sakamoto, Takashi Matsumoto, Biswajit Maiti, Kayo Okumura, Fumiki Morimatsu, G Balakrish Nair, and Hisao Kurazono. Detection of cholera toxin by an immunochromatographic test strip. In Microbial Toxins, pages 1-7. Springer, 2017.

[23] Thomas Hermann and Dinshaw J Patel. Adaptive recognition by nucleic acid aptamers. Science, 287(5454):820-825, 2000.

[24] Xiaoyu Pei, JUN Zhang, and JIE Liu. Clinical applications of nucleic acid aptamers in cancer. Molecular and clinical oncology, 2(3):341-348, 2014.

[25] Rachel Green, Andrew D Ellington, and Jack W Szostak. In vitro genetic analysis of the tetrahymena self-splicing intron. Nature, 347(6291):406-408, 1990.

[26] Craig Tuerk and Larry Gold. Systematic evolution of ligands by exponential enrichment: Rna ligands to bacteriophage t4 dna polymerase. science, 249(4968):505-510, 1990.

[27] Kuo He, Qingwen Mao, Xiuyuan Zang, Yanyu Zhang, Hui Li, and Donghao Zhang. Production of a broad-specificity monoclonal antibody and application as a receptor to detection amatoxins in mushroom. Biologicals, 49:57-61, 2017.

[28] Michael Famulok, Guenter Mayer, and Michael Blind. Nucleic acid aptamers from selection in vitro to applications in vivo. Accounts of Chemical research, 33(9):591-599, 2000.

[29] Francesca Torrini, Pasquale Palladino, Alvaro Brittoli, Veronica Baldoneschi, Maria Minunni, and Simona Scarano. Characterization of troponin t binding aptamers for an innovative enzyme-linked oligonucleotide assay (elona). Analytical and bioanalytical chemistry, 411(29):7709-7716, 2019.

[30] Miriam Jauset-Rubio, Markéta Svobodová, Teresa Mairal, Calum McNeil, Neil Keegan, Mohammad S El-Shahawi, Abdulaziz S Bashammakh, Abdulrahman O Alyoubi, and Ciara K O'Sullivan. Aptamer lateral flow assays for ultrasensitive detection of $\beta$-conglutin combining recombinase polymerase amplification and tailed primers. Analytical chemistry, 88(21):10701-10709, 2016.

[31] Shima Dalirirad and Andrew J Steckl. Lateral flow assay using aptamer-based sensing for on-site detection of dopamine in urine. Analytical biochemistry, 596:113637, 2020.

[32] Birui Jin, Yexin Yang, Rongyan He, Yong Il Park, Aeju Lee, Dan Bai, Fei Li, Tian Jian Lu, Feng $\mathrm{Xu}$, and Min Lin. Lateral flow aptamer assay integrated smartphone-based portable device for simultaneous detection of multiple targets using upconversion nanoparticles. Sensors and Actuators B: Chemical, 276:48-56, 2018.

[33] B Busra Tasbasi, Buket C Guner, Mert Sudagidan, Samet Ucak, Murat Kavruk, and Veli C Ozalp. Label-free lateral flow assay for listeria monocytogenes by aptamer-gated release of signal molecules. Analytical biochemistry, 587:113449, 2019.

[34] Ngoc Linh Phung, Johanna G Walter, Rebecca Jonczyk, Lisa K Seiler, Thomas Scheper, and Cornelia Blume. Development of an aptamer-based lateral flow assay for the detection of c-reactive protein using microarray technology as a prescreening platform. ACS Combinatorial Science, 22(11):617-629, 2020 .

[35] Qiang Yu, Qu Zhao, Sai Wang, Shuai Zhao, Shan Zhang, Yingai Yin, and Yiyang Dong. Development of a lateral flow aptamer assay strip for facile identification of theranostic exosomes isolated from human lung carcinoma cells. Analytical biochemistry, 594:113591, 2020.

[36] Pranav Tripathi, Anand Kumar, Manisha Sachan, Sameer Gupta, and Seema Nara. Aptamergold nanozyme based competitive lateral flow assay for rapid detection of ca125 in human serum. Biosensors and Bioelectronics, 165:112368, 2020. 
[37] Shima Dalirirad and Andrew J Steckl. Aptamer-based lateral flow assay for point of care cortisol detection in sweat. Sensors and Actuators B: Chemical, 283:79-86, 2019.

[38] Jing Zhang, Xuefei Lv, Wei Feng, Xiaoqiong Li, Kunjie Li, and Yulin Deng. Aptamer-based fluorometric lateral flow assay for creatine kinase mb. Microchimica Acta, 185(8):1-8, 2018.

[39] Velu Ranganathan, Sathya Srinivasan, Aryan Singh, and Maria C DeRosa. An aptamer-based colorimetric lateral flow assay for the detection of human epidermal growth factor receptor 2 (her2). Analytical biochemistry, 588:113471, 2020.

[40] Guangyu Shen, Songbai Zhang, and Xia Hu. Signal enhancement in a lateral flow immunoassay based on dual gold nanoparticle conjugates. Clinical biochemistry, 46(16-17):1734-1738, 2013.

[41] John G Bruno. Application of dna aptamers and quantum dots to lateral flow test strips for detection of foodborne pathogens with improved sensitivity versus colloidal gold. Pathogens, 3(2):341-355, 2014.

[42] Libing Wang, Wenwei Ma, Wei Chen, Liqiang Liu, Wei Ma, Yingyue Zhu, Liguang Xu, Hua Kuang, and Chuanlai Xu. An aptamer-based chromatographic strip assay for sensitive toxin semiquantitative detection. Biosensors and Bioelectronics, 26(6):3059-3062, 2011.

[43] Klaudia Arciszewska, Dominika Bartnicka, Filip Bartnicki, Ewa Kowalska, Małgorzata BodaszewskaLubaś, Paweł Hermanowicz, Heinz Faulstich, and Wojciech Strzałka. Selection and analysis of a dna aptamer binding \alpha-amanitin from amanita phalloides. Acta Biochimica Polonica, 64(3), 2017.

[44] Candace S Bever, Bogdan Barnych, Robert Hnasko, Luisa W Cheng, and Larry H Stanker. A new conjugation method used for the development of an immunoassay for the detection of amanitin, a deadly mushroom toxin. Toxins, 10(7):265, 2018. 